\title{
A new lattice thermal conductivity model of a thin-film semiconductor
}

\author{
Mei-Jiau Huang ${ }^{\text {a }}$, Tai-Ming Chang ${ }^{\mathrm{a}, *}$, Wen-Yen Chong ${ }^{\mathrm{a}}$, Chun-Kai Liu ${ }^{\mathrm{b}}$, Chih-Kuang Yu ${ }^{\mathrm{b}}$ \\ ${ }^{a}$ Department of Mechanical Engineering, National Taiwan University, Taipei, Taiwan, ROC \\ ${ }^{\mathrm{b}}$ Electronics and Optoelectronics Research Laboratories, Industrial Technology Research Institute, Taiwan, ROC
}

Received 2 March 2006; received in revised form 12 June 2006

Available online 29 September 2006

\begin{abstract}
In the present study, a new lattice thermal conductivity model for a thin-film semiconductor is proposed. This model is considered, compared to the existing models, to be more mathematically consistent in the sense that the heat flow is contributed solely by the lowdimensional phonons, and the spatial confinement effects not only on the phonon group and phase velocities but also on the Debye temperature are taken into consideration. To count the boundary scattering effect, an analytical or empirical boundary scattering rate is suggested and added to the total scattering rate via the Mattiessen's rule. It is found this newly proposed model predicts as well as the existing models and reasonably well with the experimental data.
\end{abstract}

(C) 2006 Elsevier Ltd. All rights reserved.

Keywords: Confined dispersion relations; Low-dimensional wave number; Boundary scattering; Debye temperature

\section{Introduction}

The thermoelectric coolers have recently received a lot of attention because they have many advantages over the conventional compressors. These advantages include miniaturization, low noise, high reliability, no use of the refrigerants, and so on [1,2]. However, their practical applications are still limited because of the associated poor cooling efficiency, low cooling ability, and high cooling temperature. The performance of a thermoelectric cooler is mainly dominated by the material figure-of-merit $Z T=S^{2} \sigma T /\left(k_{1}+k_{\mathrm{e}}\right)$, where $S$ is the Seebeck coefficient, $\sigma$ is the electrical conductivity, $k_{1}$ is the lattice thermal conductivity, $k_{\mathrm{e}}$ is the electronic thermal conductivity, and $T$ is the absolute temperature [3]. Materials with high electrical conductivity (low Joule's heat generation rate) and low thermal conductivity (low Fourier's heat conduction rate) are therefore preferred. However, for the commonly known materials, those having high electric conductivities also have high thermal conductivities. Conventional thermo-

\footnotetext{
${ }^{*}$ Corresponding author. Tel.: +88623366 2762; fax: +8862 23631755 . E-mail address: d94522009@ntu.edu.tw (T.-M. Chang).
}

electric materials thus have a $Z T$ value only about one at room temperature [4].

Recently, a large enhancement of the $Z T$ values has been reported experimentally as well as theoretically [5-9] by adopting semiconductor quantum wells, quantum wires and superlattices. It is found the lattice thermal conductivity $k_{1}$ of these low-dimensional semiconductors is terribly small compared to their bulk structures due to the spatial confinement effect. The spatial confinement effect changes the energy bands (formation of minibands in superlattices), modifies the phonon dispersion relation, and increases the phonon-boundary (or interface) scattering [8,9]. Lots of studies have therefore been performed on an investigation of the phonon behavior in the semiconductor under the size effects.

In the literatures, most lattice thermal conductivity models are constructed based on the phonon Boltzmann transport equation (PBTE) under the relaxation-time approximation. According to the classification made by Zou and Balandin [10], these models can be distinguished into three types. The first one solves the PBTE as if there were no boundaries but takes into account the modifications of the phonon dispersion relation by the spatial 
confinement effect and adds a boundary scattering rate $\left(1 / \tau_{\mathrm{B}}\right)$ to the total scattering rate through the Mattiessen's rule. Balandin and Wang [11] so computed the lateral lattice thermal conductivity of a quantum well with freesurface boundaries. They found the changes of the phonon dispersion relation and of the group velocity lead to a significant increase of the scattering strength, including the Umklapp-process scattering (U), the mass-difference scattering $(\mathrm{M})$, and the boundary scattering (B). The lattice thermal conductivity is consequently reduced by more than one order in magnitude. Moreover, a weak dependence of the lattice thermal conductivity on the temperature is found, which agrees with the experimental observation [12]. Khitun et al. [13] presented a similar analysis on freeand clamped-surface quantum wires. They found that the reduction of the lattice thermal conductivity associated with a quantum wire is even much more than that associated with a quantum well.

The thermal conductivity models of the second type look for an exact solution of the PBTE with partially diffuse boundaries. Such a phonon distribution results in a modified formula for the lattice thermal conductivity. These models however adopt the bulk dispersion relation. Accepting these ideas, Walkauskas et al. [14] also discovered the reduction of the lattice thermal conductivity of GaAs quantum wells and wires. The third approach for the lattice thermal conductivity is proposed by Zou and Balandin [10] and is a combination of the former two approaches. That is, both the modified phonon dispersion relation and the modified lattice thermal conductivity formula are taken into consideration. Nonetheless, the boundary scattering effect was carelessly doubly counted in their work, once in solving the non-equilibrium phonon distribution and the other in adding a boundary scattering rate to the total scattering rate via the Mattiessen's rule.

Although the lattice thermal conductivity models mentioned above agree more or less with the experimental measurements, there exist some inconsistent assumptions in these models. The major one involves the use of a population-weighted average phonon dispersion relation. It is known, under the spatial confinement effect, there are discrete and infinitely many modes (frequencies) of phonons for a given low-dimensional wave number [15]. An imaged energy level system is usually assumed among modes having a same frequency, and based on it the equilibrium phonon populations are calculated. An average group velocity over these modes weighted by their phonon populations is thus obtained $[10,11,13]$. When computing the heat flow rate, this average phonon dispersion relation is furthermore assumed to be three-dimensionally isotropic. The irrationality obviously exists in the imaged energy level system (phonons have a same frequency but different populations), in changing the low-dimensional wave numbers into three-dimensional ones, and in turning anisotropy to isotropy. Other minor inconsistent assumptions made in the existing lattice thermal conductivity models still include a constant (average) phonon group velocity, a same Debye temperature for bulk and low-dimensional materials, and so on.

These major and minor irrational simplifications inspire the motivation of the present study, to develop a new lattice thermal conductivity model which is mathematically consistent as much as possible. A model that counts directly the contributions of each branch of phonon modes and insists on the low-dimensional wave numbers will be targeted. The boundary scattering will be viewed as a microscopic phenomenon and modeled through a characteristic relaxation time. The rest of this paper is organized as follows. In Section 2, we present the proposed lattice thermal conductivity model for semiconductor thin film, including an analytical formula for evaluating the boundary scattering rate. A silicon thin film is employed for illustration. Comparison with existing models and with the experimental measurements is given in Section 3. So are the discussions. The conclusions are given at last in Section 4.

\section{Mathematical model}

In the following subsections, we describe one by one the mathematical models for the phonon dispersion relations, the Debye temperatures, the non-equilibrium phonon distribution, the heat flux, and the scattering rates.

\subsection{Phonon dispersion relations}

Due to the low group velocity of the optic phonons, the thermal conductivities of semiconductor materials are mainly contributed by the acoustic phonons $[6,16]$. We consider the acoustic modes in an isotropic continuum material. The lattice displacement, $u_{i}$, is governed by the elasticity equation, [15]

$\frac{\partial^{2} u_{i}}{\partial t^{2}}=v_{\mathrm{t}}^{2} \nabla^{2} u_{i}+\left(v_{1}^{2}-v_{\mathrm{t}}^{2}\right) \frac{\partial^{2} u_{j}}{\partial x_{i} \partial x_{j}}$

For bulk materials, Eq. (1) is solved with a use of the threedimensional Fourier transform. The allowed vibration eigenmodes (polarizations) and the corresponding eigenvalues (frequencies) are analyzed then. The dispersion relation describing the relation between the three-dimensional wave vector $(\vec{q})$ and the frequency $\omega$ is desired. The dispersion relations of three polarizations for bulk materials are found to be: $\omega=v_{1} q$ for one longitudinal wave and $\omega=v_{\mathrm{t}} q$ for two transverse waves, where $q$ is the threedimensional wave number $\left(q^{2}=q_{x}^{2}+q_{y}^{2}+q_{z}^{2}\right)$. The longitudinal and transverse group velocities $(\mathrm{d} \omega / \mathrm{d} q)$ of bulk materials are thus constant $\left(v_{1}\right.$ and $\left.v_{\mathrm{t}}\right)$ and as same as the phase velocities $(\omega / q)$ [17]. On the other hand, if a freestanding thin-film semiconductor is considered, a plane Fourier transform is employed instead and the free-stress boundary conditions are applied in the thickness direction. Three different types of the confined phonon polarizations have also been found [15]: the shear waves, the dilatational 
waves, and the flexural waves, which are characterized by their distinctive symmetries. The dispersion relations of shear waves are given by

$\omega_{n}^{2}=v_{\mathrm{t}}^{2}\left(q_{z, n}^{2}+q^{2}\right)$

where $q_{z, n}=n \pi / a$ ( $n$ integer) is the quantized wave vector in $z$ (thickness)-direction, $a$ is the film thickness, and $q$ is now the plane wave number $\left(q^{2}=q_{x}^{2}+q_{y}^{2}\right)$. Several modes of the shear wave are shown in Fig. 1 for illustration. As seen, the first mode $(n=1)$ has a group velocity equal to $v_{\mathrm{t}}$ but higher modes have much smaller group velocities at low dimensionless wave numbers $(q a)$.

As far as the dilatational and flexural waves are considered, the dispersion relations are

$\omega^{2}=v_{1}^{2}\left(q_{1}^{2}+q^{2}\right)=v_{\mathrm{t}}^{2}\left(q_{\mathrm{t}}^{2}+q^{2}\right)$

where $q_{1}$ and $q_{\mathrm{t}}$ are determined by

$\frac{\tan \left(q_{\mathrm{t}} a / 2\right)}{\tan \left(q_{1} a / 2\right)}=-\frac{4 q^{2} q_{1} q_{\mathrm{t}}}{\left(q^{2}-q_{\mathrm{t}}^{2}\right)^{2}}$

for the dilatational waves, and by

$\frac{\tan \left(q_{1} a / 2\right)}{\tan \left(q_{\mathrm{t}} a / 2\right)}=-\frac{4 q^{2} q_{1} q_{\mathrm{t}}}{\left(q^{2}-q_{\mathrm{t}}^{2}\right)^{2}}$

for the flexural waves [15]. It is known that either Eq. (4) or Eq. (5) has discrete and infinitely many solutions for a given wave number $q$ as shown in Fig. 2. As seen, all modes have group velocities negligible at small wave numbers, close to the bulk longitudinal one at intermediate wave numbers, and transiting to the bulk transverse one at very large wave numbers, except the first mode which displays only the latter two.

In order to handle the discrete and infinitely many modes of phonons, a population-weighted average group velocity [11] or an algebraic averaged one [18] has been proposed. The average dispersion relation is then used as if it were a three-dimensionally isotropic one ( $q$ is automatically changed to be the three-dimensional wave number), when the heat flow rate is evaluated. Figs. 1 and 2 however

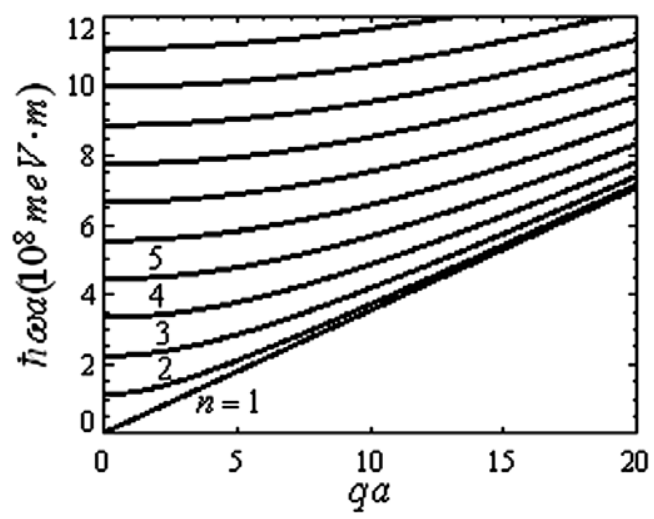

Fig. 1. The phonon modes of the shear waves in the silicon thin film. The transverse polarization of the bulk dispersion relations coincides with the lowest phonon mode.

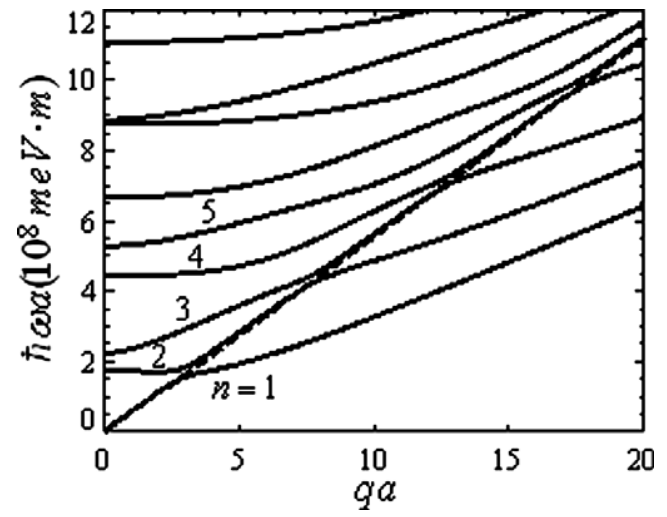

Fig. 2. The phonon modes of the dilatational waves in the silicon thin film. The dash line is the longitudinal polarization of the bulk dispersion relations.

show that all modes of three polarizations have group velocities convergent to the bulk transverse velocity at very large $q a$. In other words, all three polarizations converge to the bulk transverse polarization as $a \rightarrow \infty$. The bulk thermal conductivity cannot be recovered therefore. In this study, we thus give up the averaging method but count directly the contributions of all phonon modes of all polarizations. Most of all, we insist that the wave number $q$ must remain two-dimensional consistently.

\subsection{Debye temperature}

In the previous investigations, a same Debye temperature is usually employed for both bulk and low-dimensional materials. Nonetheless, the Debye temperature may also be changed by the spatial confinement effect. According to the Debye model [19-21], the maximum wave number $q_{\max }$ associated with phonons in the isotropic crystal of a diamond structure such as silicon can be calculated as follows

$\frac{4 \pi}{3} q_{\max }^{3}=4 \frac{(2 \pi)^{3}}{a_{0}^{3}}$

where $a_{0}$ is the lattice constant. Taking this maximum wave number and using the dispersion relations determined by Eqs. (2)-(5), one can find the cut-off frequency $\omega_{\mathrm{D}}\left(q_{\max }\right)$ and thus determine the Debye temperature $\theta_{\mathrm{D}}=\hbar \omega_{\mathrm{D}} /$ $k_{\mathrm{B}} T$ for phonons of each mode of each polarization.

\subsection{Non-equilibrium phonon distribution}

We adopt as usual the steady relaxation-time-approximated phonon Boltzmann equation [16,20] as follows

$\vec{v} \cdot \nabla \tilde{n}+\frac{\tilde{n}}{\tau}=\vec{v} \cdot \nabla n_{0}$

where $n=n_{0}-\tilde{n}$ is the phonon distribution function, $\vec{v}$ is the phonon group velocity, $1 / \tau$ is the total scattering rate, and $n_{0}$ is the equilibrium phonon distribution at the local temperature $T$, namely the Bose-Einstein distribution [21], 
$n_{0}=\frac{1}{\exp \left(\hbar \omega / k_{\mathrm{B}} T\right)-1}$

with $\hbar$ and $k_{\mathrm{B}}$ being the Planck constant and the Boltzmann constant respectively.

Now suppose that the temperature gradients exist in the $x$-direction and that the temperature gradients are so small that variations of local equilibrium distribution $n_{0}$ in space are negligible. The solution $\tilde{n}$ of Eq. (7), the non-equilibrium part of the phonon distribution, is then easily found to be

$\tilde{n}=v_{x} \tau \frac{\partial T}{\partial x} \frac{\partial n_{0}}{\partial T}$

for bulk materials, where $v_{x}$ is the $x$-component of the group velocity. It is actually also true for the free-standing thin-film semiconductor because only in-plane (low-dimensional) waves are concerned herein. Strictly speaking, phonons of a quantized mode can be no longer viewed as approaching the nanostructure surface but phonons of a superposition of modes can. The boundary scattering effect will be handled through a use of the boundary scattering rate. In solving the phonon non-equilibrium distribution and later evaluating the total heat flow rate, the wave vector remains two-dimensional as it should be.

\subsection{Heat flux}

The energy carried by phonons of frequency $\omega$ and of a population $n$ is $n \hbar \omega$. We thus propose to compute the heat flux $J_{Q}$ in the $x$-direction as

$J_{Q}=-\frac{1}{(2 \pi)^{2}} \frac{1}{a} \sum_{s} \sum_{n} \int \tilde{n} \hbar \omega v_{x} \mathrm{~d} \vec{q}$

where the summation $n$ is done over the infinitely many phonon modes and over all three polarizations $(s)$; contributions from phonons of all possible wave vectors are integrated as well $[20,21]$. Noticing only the non-equilibrium part $(\tilde{n})$ of the phonon population makes a non-zero net contribution to the heat flux, by substituting Eq. (9) into Eq. (10) and adopting the Fourier conduction law

$J_{Q}=-k_{1} \frac{\partial T}{\partial x}$

we obtain the lattice thermal conductivity $\left(k_{1}\right)$ of thin film as follows

$k_{1}=\frac{1}{(2 \pi)^{2}} \frac{1}{a} \sum_{s} \sum_{n} \int \tau v_{x}^{2}(\hbar \omega) \cdot \frac{\partial n_{0}}{\partial T} \mathrm{~d} \vec{q}$

with $v_{x}=v \cos \theta$ and $\mathrm{d} \vec{q}=q \mathrm{~d} q \mathrm{~d} \theta$, Eq. (12) can be rewritten as

$k_{1}=\frac{1}{4 \pi a} \frac{k_{\mathrm{B}}^{3} T^{2}}{\hbar^{2}} \sum_{s} \sum_{n} \int_{x_{0}}^{\theta_{\mathrm{D}} / T} \tau v \frac{x^{3} \mathrm{e}^{x}}{\left(\mathrm{e}^{x}-1\right)^{2}} \cdot \frac{q}{\omega} \mathrm{d} x$

where $x=\hbar \omega / k_{\mathrm{B}} T$ and the integration limits $\left(x_{0}\right.$ and $\left.\theta_{\mathrm{D}} / T\right)$ are the minimum and maximum dimensionless frequencies of each curve in Figs. 1 and 2 for $0 \leqslant q \leqslant q_{\max }$. They are different for different phonon modes therefore. In all the calculations below, the integration is performed nonetheless over the wave number instead, because it is easier and because some phonon modes do not have a unique inverse dispersion relation $q(\omega)$.

It is worth mentioning that in the previous models $[10,13]$, the thermal conductivity of low-dimensional materials is often written as $k_{1}-\Delta k$, where $\Delta k$ arises from the collisions between phonons and the boundaries. In deriving the phonon dispersion relation of a free-standing thin film, only waves traveling in the plane are allowed however. It seems then no phonons can collide with the boundaries. This is not true because the associated amplitudes of these in-plane waves are strongly affected by the boundaries. To model this effect, we explain the plane-traveling waves as a lumped result. The boundary scattering instead is viewed as a microscopic phenomenon and may be modeled through a use of a characteristic relaxation time $\left(\tau_{\mathrm{B}}\right)$ as introduced in the following subsection.

\subsection{Relaxation time}

In the present study, we reconsider the four scattering mechanisms that were investigated by Zou and Balandin [10]. They are the Umklapp scattering $\left(1 / \tau_{U}\right)$, the massdifference scattering $\left(1 / \tau_{\mathrm{M}}\right)$, the phonon-electron scattering $\left(1 / \tau_{\mathrm{Ph}-\mathrm{e}}\right)$, and the boundary scattering $\left(1 / \tau_{\mathrm{B}}\right)$. For a detailed description of the former three, the readers are referred to the work of Zou and Balandin [10]. In the followings, we introduce the boundary scattering rate $\left(1 / \tau_{\mathrm{B}}\right)$ employed in the present study.

We first recall that when the phonon group velocities are considered to be three-dimensional, phonons collide with boundaries. The resulting lattice thermal conductivity becomes $k_{1}-\Delta k[14,19]$, where

$k_{1}=\frac{1}{6 \pi^{2}} \frac{k_{\mathrm{B}}^{4} T^{3}}{\hbar^{3}} \sum_{s} \int_{0}^{\theta_{\mathrm{D}} / T} \tau v \cdot \frac{x^{4} \mathrm{e}^{x}}{\left(\mathrm{e}^{x}-1\right)^{2}} \cdot \frac{q^{2}}{\omega^{2}} \mathrm{~d} x$

and

$\Delta k=\frac{a}{16 \pi^{2}} \frac{k_{\mathrm{B}}^{4} T^{3}}{\hbar^{3}} \sum_{s} \int_{0}^{\theta_{\mathrm{D}} / T} \frac{H(\eta)}{\eta^{2}} \frac{x^{4} \mathrm{e}^{x}}{\left(\mathrm{e}^{x}-1\right)^{2}} \cdot \frac{q^{2}}{\omega^{2}} \mathrm{~d} x$

The function $H(\eta)$ is given by

$H(\eta) \equiv(1-p)-(1-p)^{2} \sum_{i=1}^{\infty} p^{i-1} D(i \eta)$

with

$D(\eta) \equiv\left(1-\frac{5 \eta}{3}-\frac{\eta^{2}}{6}+\frac{\eta^{3}}{6}\right) \mathrm{e}^{-\eta}+2 \eta^{2}\left(1-\frac{\eta^{2}}{12}\right) \cdot \int_{\eta}^{\infty} \frac{\mathrm{e}^{-x}}{x} \mathrm{~d} x$

and

$\eta=\frac{a}{\ell}=\frac{a}{\tau v}$ 
The symbol $p$ represents the interfacial roughness and is defined as the fraction of phonons that are specularly reflected at the boundary. Special carefulness is required in evaluating the scattering rate $1 / \tau$ however. Because the boundary scattering effect has been counted through $\Delta k$, the scattering rate $1 / \tau$ should include only the other three. That is

$\frac{1}{\tau}=\frac{1}{\tau_{\mathrm{U}}}+\frac{1}{\tau_{\mathrm{M}}}+\frac{1}{\tau_{\mathrm{Ph}-\mathrm{e}}}$

We now attempt to derive a relaxation time to model the boundary scattering effect. We first write the lattice thermal conductivity of a thin-film semiconductor in a form like that suitable for bulk materials, i.e.

$k_{\text {film }}=\frac{1}{6 \pi^{2}} \frac{k_{\mathrm{B}}^{4} T^{3}}{\hbar^{3}} \sum_{s} \int_{0}^{\theta_{\mathrm{D}} / T} \tau^{\prime} v \cdot \frac{x^{4} \mathrm{e}^{x}}{\left(\mathrm{e}^{x}-1\right)^{2}} \cdot \frac{q^{2}}{\omega^{2}} \mathrm{~d} x$

Comparing Eq. (20) with Eqs. (14) and (15), we find

$\tau^{\prime}=\tau\left(1-\frac{3}{8} \frac{H(\eta)}{\eta}\right)$

According to the Mattiessen's rule, we may define

$\frac{1}{\tau_{\mathrm{B}}}=\frac{1}{\tau^{\prime}}-\frac{1}{\tau}$

It is easy to check that $1 / \tau_{\mathrm{B}}=0$ when $p=1$ and $1 / \tau_{\mathrm{B}} \propto v / a$ when $p=0$. In Fig. 3, we show the ratio of the so-obtained boundary scattering rate to the sum of the other three, namely $\tau_{\mathrm{B}}^{-1} / \tau^{-1}$, when $p=0$. An important property associated with this boundary scattering rate is it has a nonzero value only if some other scattering mechanisms exist. Shown in Fig. 4 are the proposed boundary scattering rates for several lowest phonon modes of the dilatational polarization in a silicon thin film of thickness $20 \mathrm{~nm}$.

Compared to the commonly used empirical boundary scattering rate

$\frac{1}{\tau_{\mathrm{B}}}=\frac{v}{a}(1-p)$

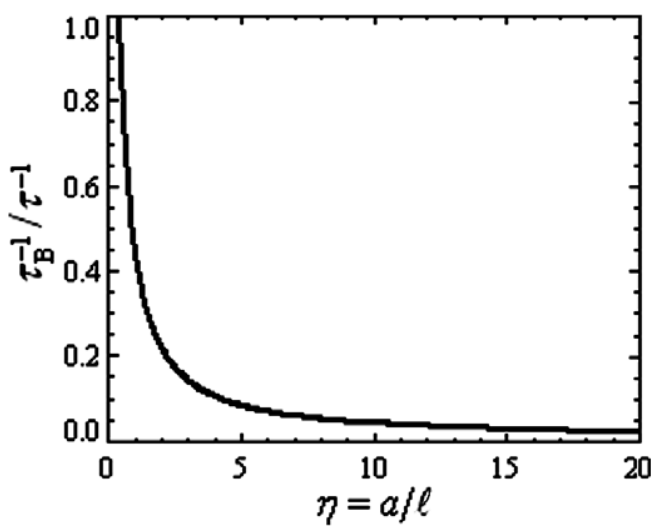

Fig. 3. The ratio of the boundary scattering rate to the sum of the others against the dimensionless film thickness when $p=0$.

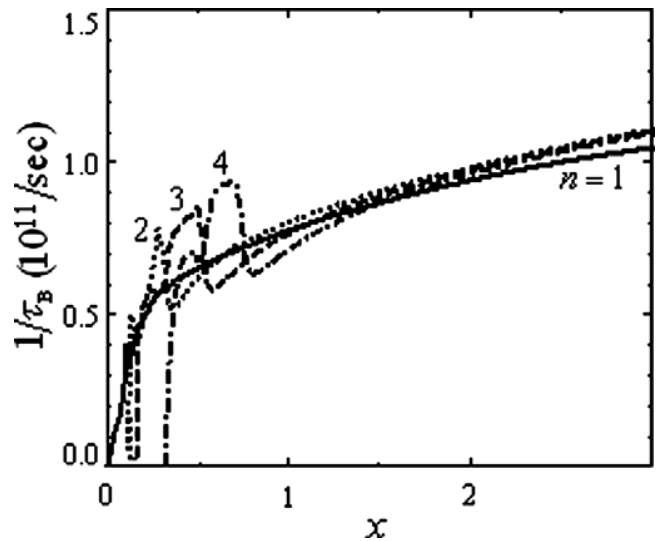

Fig. 4. The proposed boundary scattering rates of the four lowest phonon modes of the dilatational wave in the silicon thin film of thickness $20 \mathrm{~nm}$ against the non-dimensional phonon energy $(p=0)$.

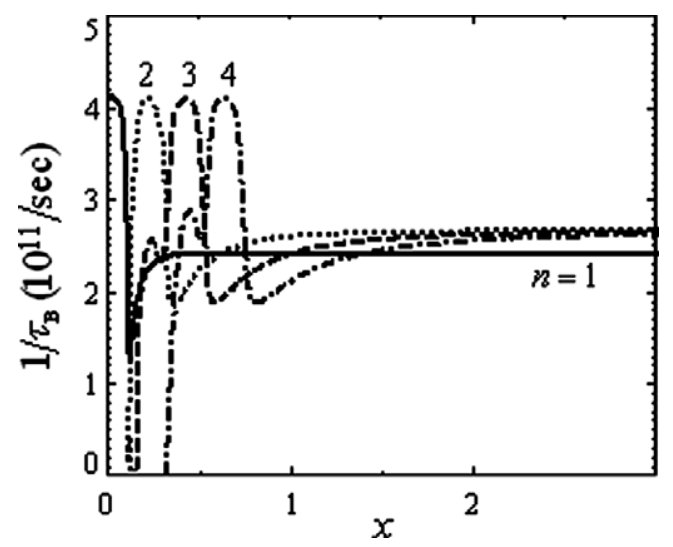

Fig. 5. The empirical boundary scattering rates of the four lowest phonon modes of the dilatational wave in the silicon thin film of thickness $20 \mathrm{~nm}$ against the non-dimensional phonon energy $(p=0)$.

as shown in Fig. 5, the newly proposed rate is less than half of the empirical one. Moreover, the newly proposed boundary scattering rates of all modes are small at small frequencies because $1 / \tau$ is small there. The empirical one of the first mode however is the largest among all.

In remark, we attempt to compute the lattice thermal conductivity of a free-standing silicon thin film in the present study by Eq. (13) (with $\tau$ replaced by $\tau^{\prime}$ ), the dispersion relations Eqs. (2)-(5), the maximum wave number Eq. (6), and the relaxation time evaluated by Eqs. (19) and (21).

\section{Results and discussion}

The lattice thermal conductivity of a silicon thin film is investigated herein for illustration. The mass density $\rho$ of silicon is $2.33 \mathrm{~kg} / \mathrm{m}^{3}$. The bulk longitudinal and transverse velocities are $v_{1}=8470 \mathrm{~m} / \mathrm{s}$ and $v_{\mathrm{t}}=5340 \mathrm{~m} / \mathrm{s}$ respectively. All other values of material properties and model parameters are extracted directly from the work of Zou and Balandin [10]. With a lattice constant of $5.43 \AA$, the maximum wave number of silicon is found to be about $11.4 \mathrm{~nm}^{-1}$, 
according to Eq. (6). The Debye temperatures in Eq. (13) are then determined by using this maximum wave number and the dispersion curves in Figs. 1 and 2. They are therefore different from mode to mode and from polarization to polarization.

In Figs. 6 and 7, we show the contribution to the lattice thermal conductivity from each phonon mode of the dilatational and shear waves. The temperature is $300 \mathrm{~K}$ and the boundary is purely diffuse $(p=0)$. Several interesting phenomena are observed. First, the most contribution among modes of the dilatational wave comes from the second mode instead of the first one. Although the first mode has larger group velocity $(\mathrm{d} \omega / \mathrm{d} q)$ and smaller phase velocity $(\omega / q)$, its Debye temperature is smaller. Secondly, there are some intermediate modes of the dilatational wave which contributions to the lattice thermal conductivity are approximately equal and non-negligible. These modes are those that possess a subrange of wave numbers having group velocity close to $v_{1}$ and below the maximum wave number. For even higher modes, their contributions are less and decrease rapidly with increasing $n$. Unlike the dilatational wave, the contribution curves of the shear wave in Fig. 7 decrease smoothly and rapidly with increasing $n$.

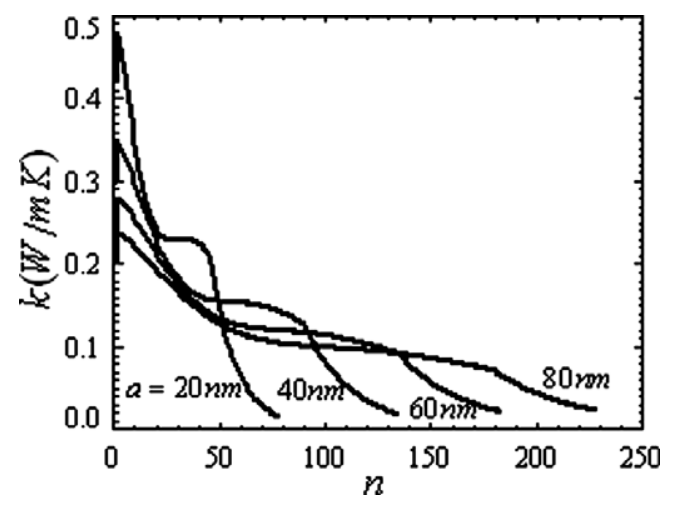

Fig. 6. Contributions to the lattice thermal conductivity from the phonon modes of the dilatational waves in the silicon thin film of thickness $20 \mathrm{~nm}$ at $T=300 \mathrm{~K}(p=0)$.

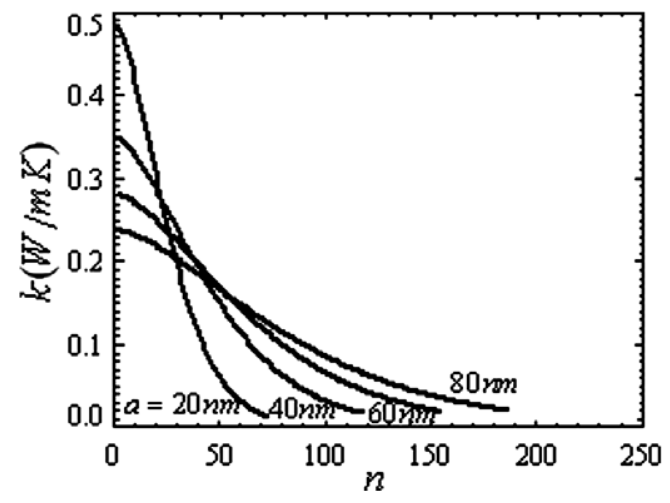

Fig. 7. Contributions to the lattice thermal conductivity from the phonon modes of the shear waves in the silicon thin film of thickness $20 \mathrm{~nm}$ at $T=300 \mathrm{~K}(p=0)$.
Finally, from both figures, one notices that the number of phonon modes required for a converged total lattice thermal conductivity increases with increasing film thickness. This is because when the film is thicker ( $q_{\max } a$ larger $)$, there are more and more modes possessing the subrange of wave numbers having group velocity close to $v_{1}$. When the film is very thick, tremendously many modes must be required and a bulk thermal conductivity value should be obtained. In the following calculations, we count the contributions of all modes up to the one that contributes less than $0.1 \%$, that is

$k_{n} / \sum_{i=1}^{n} k_{i}<0.1 \%$

The number of modes used against the film thickness is shown in Fig. 8. Basically, several hundreds of modes are required for a thin film of thickness from a few tens to a few hundreds of nanometers.

In Fig. 9 we show the computed lattice thermal conductivity of the thin film against the temperature. The film

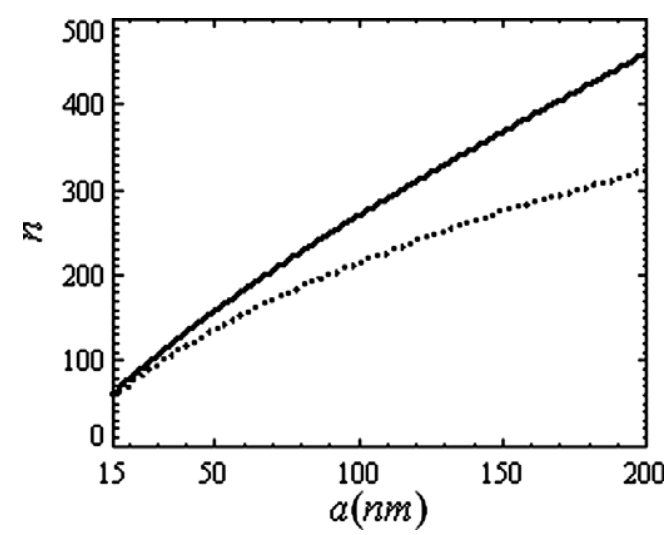

Fig. 8. The number of phonon modes required for a convergence of the lattice thermal conductivity against the film thickness; solid line: dilatational waves; dot line: shear waves.

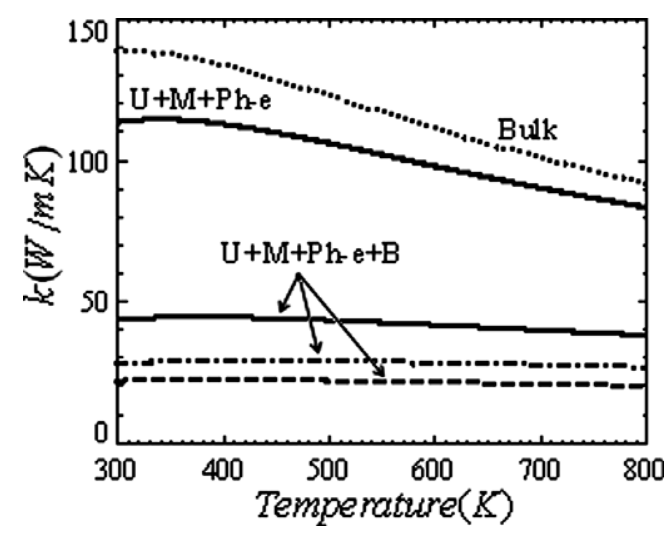

Fig. 9. The lattice thermal conductivity of the silicon thin film of thickness $20 \mathrm{~nm}$ against the temperature $(p=0)$. The solid and the dash-doted curves are predicted by using the analytical and the empirical boundary scattering rates respectively; the dash curve is predicted by the A1 model. 
thickness is $20 \mathrm{~nm}$ and the boundary is purely diffuse $(p=0)$. It is seen scattering due to the Umklapp processes, the mass-difference, and the phonon-electron interactions causes only a little reduction of the lattice thermal conductivity. A huge reduction is observed however when the boundary scattering is included. And it is found also the lattice thermal conductivity depends weakly on temperature, agreeing with the experimental measurements [12]. Also shown in Fig. 9 is the prediction based on the socalled A1 model proposed by Zou and Balandin [10], in which a population-averaged phonon dispersion relation of the dilatation wave is employed for all polarizations, the lattice thermal conductivity formula Eq. (14) with $\tau$ being replaced by $\tau^{\prime}$ is adopted, the empirical boundary scattering rate Eq. (23) is taken, and finally the bulk Debye temperature $625 \mathrm{~K}$ is used. The lattice thermal conductivity predicted by the present model is close to but larger than that predicted by the A1 model, even in use of the empirical boundary scattering rate. It is mainly because the Debye temperatures employed in the present model are higher. Although the prediction by the A1 model is closer to the experimental measurement [23] (22 W/m K at $300 \mathrm{~K})$, an extrapolation of the measurements by $\mathrm{Ju}$ and Goodson [22] nonetheless suggests a value of $46 \mathrm{~W} / \mathrm{m} \mathrm{K}$. Anywise, the newly proposed model is considered to be more mathematically consistent.

Shown in Fig. 10 is the lattice thermal conductivity of the silicon thin film against the interfacial roughness, namely the specular fraction $p$. The lattice thermal conductivity decreases with increasing roughness (decreasing $p$ ) as expected. Interestingly, the A1 model predicts a same lattice thermal conductivity as the present model does when $p=0$. The value however is still less than the bulk counterpart because of the employed confined dispersion relations. At last, we calculate the lattice thermal conductivity against the film thickness and compare it with the experimental measurements $[22,23]$ in Fig. 11. A good agreement, except the film of thickness $20 \mathrm{~nm}$, is observed, although

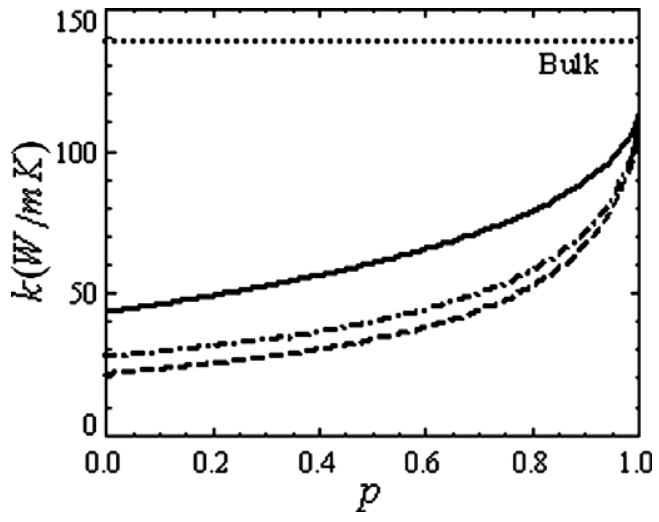

Fig. 10. The lattice thermal conductivity of the silicon thin film of thickness $20 \mathrm{~nm}$ against the fraction of boundary specular reflection at $T=300 \mathrm{~K}$. The solid and the dash-dotted curves are predicted by using the analytical and the empirical boundary scattering rates respectively; the dash curve is predicted by the A1 model.

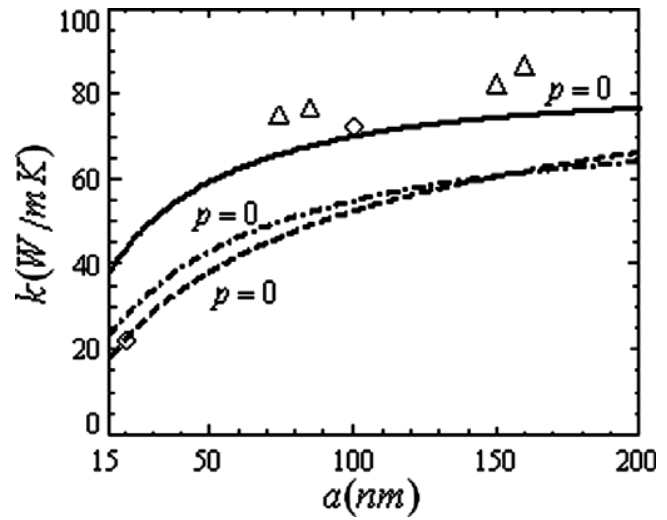

Fig. 11. A comparison of the computed lattice thermal conductivity of the silicon thin film at $T=300 \mathrm{~K}$ with the experimental measurements by $\mathrm{Ju}$ and Goodson [22] $(\triangle)$ and Liu and Asheghi [23] $(\diamond)$. The solid and the dash-dotted curves are predicted by using the analytical and the empirical boundary scattering rates respectively; the dash curve is predicted by the A1 model.

the specular fraction $p$ works as a free parameter and can be adjusted to fit the measurements. The disagreement between the predicted (from the present model) and measured thermal conductivities of a silicon thin film of thickness $20 \mathrm{~nm}$ may not be real, because the variance associated with the measurement of Liu and Asheghi [23] is as high as $\pm 20 \mathrm{~W} / \mathrm{m} \mathrm{K}$. As mentioned above, an extrapolation of the measurements by $\mathrm{Ju}$ and Goodson [22] nonetheless suggests a value of $46 \mathrm{~W} / \mathrm{m} \mathrm{K}$, slightly larger than the predicted value by the present model with an analytical boundary scattering rate. Agreement regardless of the film thickness is thus obtained.

Finally, it must be mentioned when the film thickness is small relative to the phonon wave length, the wave properties of phonons must be taken into account for an accurate evaluation of the thermal conductivity [24]. The dominant phonon wavelength at $300 \mathrm{~K}$ is about $10 \mathrm{~nm}$ [23], smaller than the thickness of the silicon films under investigation.

\section{Conclusion}

In the present study, a lattice thermal conductivity model for a thin-film semiconductor is developed in the way that the integration appearing in the lattice thermal conductivity formula is done over the low-dimensional wave numbers and all possible spatial confinement effects are counted. Instead of using a population-weighted dispersion relation like previous models, the maximum wave number is first determined by the material lattice constant, and contributions to the thermal conductivity from phonons having wave numbers less than this maximum wave number, of different modes and of different polarizations, are calculated individually and added together. Finally, to count the boundary scattering effect, an analytical or empirical boundary scattering rate is suggested and added to the total scattering rate via the Mattiessen's rule. 
Silicon thin film is investigated for example. The calculations show that a few hundreds of modes for each polarization are required in evaluating the lattice thermal conductivity of a thin film of thickness 10-200 nm. Particularly when the confined polarizations are concerned, many modes possess phonons moving at group velocities close to the bulk longitudinal velocity and making nonnegligible contributions to the thermal conductivity. The thicker the film, the more these modes are there. With many enough modes counted, this newly proposed model predicts as well as previous models do and reasonably well with the experimental data. Emphasized nonetheless is its consistency in handling all the spatial confinement effects. The model can be easily applied to other semiconductor thin films as long as fundamental material properties are available, such as the bulk longitudinal and transverse velocities as well as the lattice constant, and all the associated scattering rates are properly estimated. The latter is a complicated and difficult task and beyond the scope of the present study.

\section{Acknowledgements}

The support of this work by the National Science Council, Taiwan, ROC under contract NSC 94-2212-E-002-046 and that by ITRI under the contract 0000108475 are gratefully acknowledged.

\section{References}

[1] M.J. Huang, R.H. Yen, A.B. Wang, The influence of the Thomson effect on the performance of a thermoelectric cooler, Int. J. Heat Mass Transfer 48 (2005) 413-418.

[2] R. Chein, G. Huang, Thermoelectric cooler application in electronic cooling, Appl. Thermal Eng. 24 (2004) 2207-2217.

[3] S. Wisniewski, B. Staniszewski, R. Szymanik, Thermodynamics of Nonequilibrium Processes, PWN-Polish Scientific Publishers, Boston, 1976.

[4] D.M. Rowe, CRC Handbook of Thermoelectrics, CRC Press LLC, 1995.

[5] L.D. Hicks, M.S. Dresselhaus, Effect of quantum-well structures on the thermoelectric figure of merit, Phys. Rev. B 47 (1993) $12727-$ 12731 .
[6] G. Chen, A. Shakouri, Heat transfer in nanostructures for solid-state energy conversion, J. Heat Transfer 124 (2002) 242-252.

[7] W.S. Capinski, M. Cardona, D.S. Katzer, H.J. Maris, K. Ploog, T. Ruf, Thermal conductivity of GaAs/AlAs superlattices, Physica B 263-264 (1999) 530-532.

[8] D.G. Cahilla, W.K. Ford, K.E. Goodson, G.D. Mahan, A. Majumdar, H.J. Maris, R. Merlin, S.R. Phillpot, Nanoscale thermal transport, J. Appl. Phys. 93 (2003) 793-818.

[9] R. Venkatasubramanian, E. Siivola, T. Colpitts, B. O'Quinn, Thinfilm thermoelectric devices with high room-temperature figures of merit, Nature 413 (2001) 597-602.

[10] J. Zou, A. Balandin, Phonon heat conduction in a semiconductor nanowire, J. Appl. Phys. 89 (2001) 2932-2938.

[11] A. Balandin, K.L. Wang, Significant decrease of the lattice thermal conductivity due to phonon confinement in a free-standing semiconductor quantum well, Phys. Rev. B 58 (1998) 1544-1549.

[12] S.-M. Lee, D.G. Cahilla, R. Venkatasubramanian, Thermal conductivity of Si-Ge superlattices, Appl. Phys. Lett. 70 (1997) 2957-2959.

[13] A. Khitun, A. Balandin, K.L. Wang, Modification of the lattice thermal conductivity in silicon quantum wires due to spatial confinement of acoustic phonons, Superlattices Microstruct. 26 (1999) 181-193.

[14] S.G. Walkauskas, D.A. Broido, K.K. Kempa, T.L. Reinecke, Lattice thermal conductivity of wires, J. Appl. Phys. 85 (1999) 2579-2582.

[15] N. Bannov, V. Aristov, V. Mitin, Electron relaxation times due to the deformation-potential interaction of electrons with confined acoustic phonons in a free-standing quantum well, Phys. Rev. B 51 (1995) 9930-9942.

[16] G. Chen, Nanoscale Energy Transport and Conversion, Oxford, New York, 2005.

[17] G.B. Whitham, Linear and Nonlinear Waves, Wiley, New York, 1974.

[18] M.J. Huang, W.Y. Chong, T.M. Chang, The lattice thermal conductivity of a semiconductor nanowire, J. Appl. Phys. 99 (11) (2006) 114318

[19] P. Hyldgaard, G.D. Mahan, Phonon Knudsen flow in GaAs/AlAs superlattices, Thermal Cond. 23 (1996) 172-182.

[20] J.M. Ziman, Electrons and Phonons, Oxford University Press, Clarendon, 2001.

[21] N.W. Ashcroft, N.D. Mermin, Solid State Physics, Brooks/Cole, 1976.

[22] Y.S. Ju, K.E. Goodson, Phonon scattering in silicon films with thickness of order 100 nm, Appl. Phys. Lett. 74 (1999) 3005-3007.

[23] W. Liu, M. Asheghi, Phonon-boundary scattering in ultrathin singlecrystal silicon layers, Appl. Phys. Lett. 84 (2004) 3819-3821.

[24] M.V. Simkin, G.D. Mahan, Minimum thermal conductivity of superlattices, Phys. Rev. Lett. 84 (2000) 927-930. 